\title{
Finanzierung von psychomotorischen Angeboten
}

Sobald ein psychomotorisches Angebot geplant, empfohlen oder grundsätzlich vorgestellt wird, ist im Erstkontakt mit Kooperationspartnern, Eltern und Studierenden häufig eine Frage zentral: "Wie wird das denn finanziert?« Wie viele andere Fragen im Themenfeld der Psychomotorik ist auch diese Frage individuell und immer eingebettet in einen Gesamtkontext zu beantworten. Hier wird daher aus langjähriger eigener Praxiserfahrung heraus ein Überblick über die verschiedenen Möglichkeiten der Finanzierung von psychomotorischen Angeboten im therapeutischen und präventiven Bereich gegeben.

\section{Finanzierung im therapeutischen Bereich}

Psychomotorische Angebote werden in Deutschland als präventive oder therapeutische bzw. heilpädagogische Maßnahme von Vereinen, Kliniken, Jugendhilfe-Einrichtungen, Frühförderstellen, Beratungsstellen und von freien Praxen angeboten und durchgeführt. Die Finanzierung der Angebote verläuft sehr individuell und kann von Bundesland zu Bundesland und von Kommune zu Kommune variieren. Sie erfolgt über verschiedene gesetzlich verankerte oder private Wege, die nachstehend skizziert werden, ohne Anspruch auf Vollständigkeit zu erheben.

\section{Eingliederungshilfe für seelisch behinderte Kinder und Jugendliche (SGB VIII KJHG §35a)}

Therapieeinrichtungen können die Kosten für psychomotorische Angebote im Rahmen der Eingliederungshilfe mit dem kommunalen Jugendamt/der Jugendhilfe abrechnen, wenn ein entsprechender Vertrag vereinbart wurde. Dies gilt für therapeutische Maßnahmen für Kinder und Jugendliche im Schulalter, sofern ihre seelische Gesundheit und hierdurch die Teilhabe am gesellschaftlichen Leben beeinträchtigt ist oder dies zu erwarten ist. Die gesetzliche Grundlage ist im SGB VIII §35a des Kinder- und JugendhilfeGesetzes (KJHG) geregelt. Mit einem diagnostizierten Befund, einer Stellungnahme des Kinderarztes und der Schule stellen die Eltern einen Antrag auf Kostenübernahme bei einem der Jugendhilfedienste, z. B. über eine wohnortnahe Erziehungsberatungsstelle, die diesen mit einer Stellungnahme an die Jugendhilfedienste weiterleiten. Eine Kopie des Bescheides über die bewilligten Therapiestunden wird der therapeutischen Einrichtung zu Beginn der Förderung vorgelegt. Eine Weiterbewilligung nach Ablauf der Therapiestunden kann bei begründetem Bedarf beantragt werden. Die Stundensätze und Leistungen werden in der Regel mit den Kommunen ausgehandelt.

\section{Eingliederungshilfe für behinderte Menschen (SGB XII $\S \S 53 \mathrm{ff})$}

Für Kinder im Vorschulalter kann eine Kostenübernahme im Rahmen der Sozialhilfe beantragt werden. Die Eltern von Kindern mit einer Behinderung, oder von Kindern, die von einer Behinderung bedroht sind, stellen einen Antrag beim Fachdienst des kommunalen Sozialamtes. Ein fundierter Entwicklungsbericht sowie eine Stellungnahme des Kinderarztes und der Kindertagesstätte werden dazu benötigt (teilweise auch eine Kostenabsage durch die gesetzliche Krankenkasse). Die gesetzliche Grundlage bietet hier das SGB XII mit den $\S \S 53,54$ und 55. Auch hier stimmen Therapieeinrichtung und Sozialamt die Stundensätze und Leistungen individuell $a b$. Für die Eltern besteht die Möglichkeit, nach Ablauf der Therapie bei Bedarf und mithilfe eines Entwicklungsberichtes eine Weiterbewilligung zu beantragen.

\section{Hilfe zur Erziehung (SGB VIII KJHG \$ 27)}

Eine einzelfallbezogene psychomotorische Förderung kann dann auf Antrag über das SGB VII KJHG §27 gewährt werden, wenn ein Kind oder ein/e Jugendliche/ $r$ unter deutlichem Einbezug der Eltern gefördert wird. Hier ist das Jugendamt zuständig, eine Begleitung der Familien erfolgt über die Ju- 
gendhilfedienste. Umfang und Kosten der Leistungen werden zwischen dem jeweiligen Träger und der Jugendhilfe vertraglich vereinbart.

\section{Rehabilitationssport (SGB IX § 44)}

Die Teilnahme an einer Psychomotorik-Gruppe kann auch über eine Verordnung des Arztes und Bezuschussung der Krankenkasse finanziert werden. Das SGB IX § 44 regelt dazu die Rehabilitation und Teilhabe behinderter Menschen. Auch Menschen, die von Behinderung bedroht sind, haben einen Anspruch auf diese Leistung. In der Regel werden 50 Einheiten à 45 Minuten ärztlich verordnet. Die Verordnung muss dann von der jeweiligen Krankenkasse genehmigt werden. Das Angebot, in der Regel als Bewegungsspiele in der Gruppe deklariert, findet in einer Gruppe mit maximal 10 Personen statt. Die Angebote müssen ärztlich betreut werden. Diese Betreuung muss aber im Bereich Psychomotorik nicht durch einen anwesenden Arzt/eine anwesende Ärztin erbracht werden. Vielmehr muss eine mögliche Konsultation gewährleistet werden. Rehabilitationsträ-

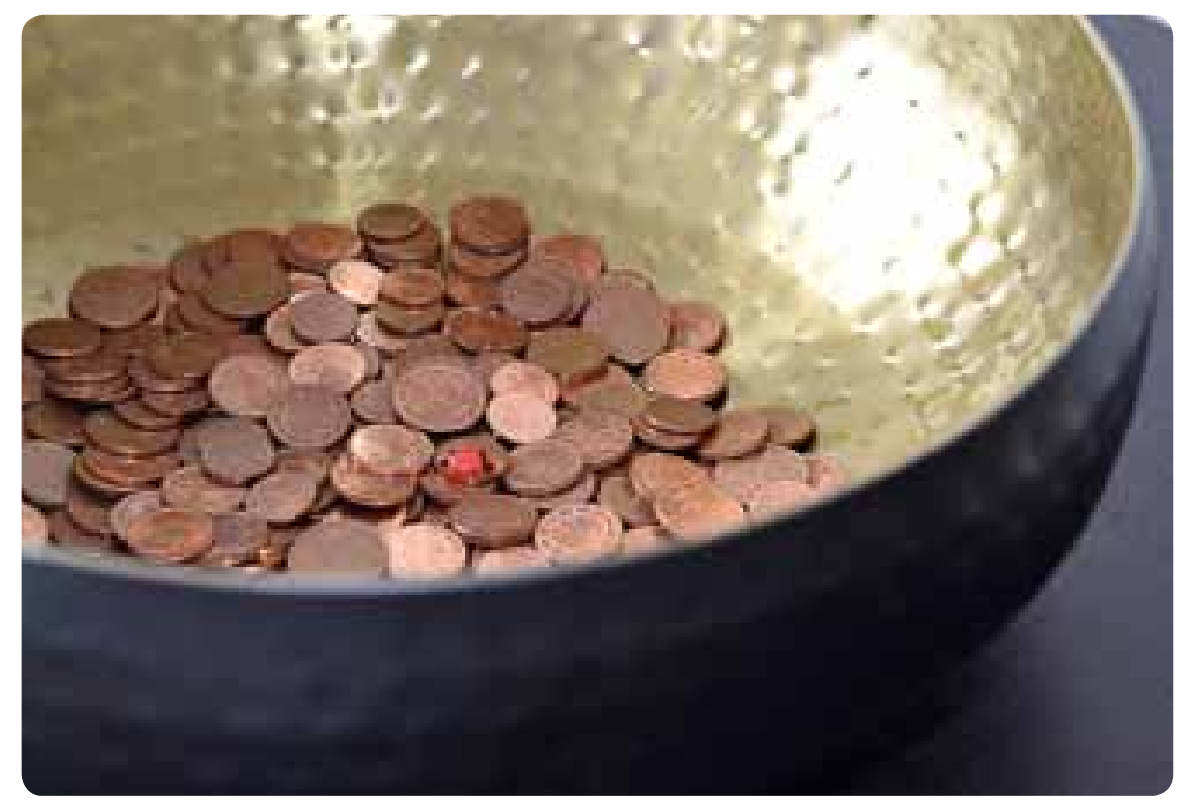

Abb. 1: Psychomotorische Angebote - auch eine Frage der Finanzierung ger sind anerkannte Sportvereine, die entsprechend ausgebildete Übungsleiterlnnen, Räumlichkeiten sowie regelmäßige Qualitätssicherungsmaßnahmen aufweisen.

\section{Privatleistungen}

Wird eine Kostenübernahme nicht bewilligt oder möchte eine Familie den Weg einer diagnostischen Abklärung und Beantragung nicht gehen, kann eine psychomotorische Förderung auch privat finanzieren werden. In diesem Fall wird der Umfang der Therapiemaßnahme individuell abgesprochen und berechnet. Im Einzelfall erstattet eine private Krankenversicherung und/oder die Beihilfe die Beiträge.

\section{Finanzierung im präventiven Bereich}

Gesundheitsförderung und Prävention - Aspekte, die für die Psychomotorik seit mehreren Jahren in Theorie und Praxis bearbeitet werden und zunehmend an Bedeutung gewinnen. Hier sind es insbesondere Vereine, Bildungseinrichtungen wie Kita und
Schule sowie freie Praxen und Freiberuflerlnnen, die Psychomotorik-Gruppen anbieten.

\section{Privatleistungen}

Die private Kostenübernahme stellt im präventiven Bereich noch den größten Anteil dar. Psychomotorik-Gruppen werden z.B. in Form von Kursen in der Kita oder im Verein durch Übungsleiterlnnen gestaltet. Eltern melden ihre Kinder entweder zu einzelnen Kursen oder als Mitglied im Verein an. Die Beiträge sind demnach sehr unterschiedlich. In Einzelfällen und auf Antrag erstatten gesetzliche und private Krankenkassen die Kursgebühr nach regelmäßiger und bescheinigter Teilnahme. Dies ist vor allem dann möglich, wenn die Angebote als Gesundheitssportangebote zertifiziert sind. Hier lohnt sich für Eltern eine Nachfrage bei der jeweiligen Krankenkasse.

\section{Projektfinanzierung und Bußgeldliste}

Das Einwerben von Projektmitteln bei Stiftungen, Bundes- und Landesinitiativen oder Fördervereinen ist vor allem dann eine Möglichkeit der Finanzierung, wenn die psychomotorischen Angebote für einen begrenzten Zeitraum und meist auch für eine spezifische Zielgruppe geplant werden. Träger sind hier gemeinnützig anerkannte Einrichtungen wie Vereine und zunehmend auch gemeinnützige Gesellschaften mit beschränkter Haftung (gGmbH). Die Anerkennung als Träger der Jugendhilfe ist in vielen Fällen die Voraussetzung, dass Stiftungsgelder und öffentliche Mittel beantragt werden können. Gleiches gilt auch für die Eintragung in die sogenannte Bußgeldliste. Gerichte und Staatsanwaltschaften weisen jährlich hohe Summen aus Bußgeldverfahren gemeinnützigen Einrichtungen zu. 
Diese können sich beim zuständigen Oberlandesgericht registrieren lassen und regelmäßig über ihre Tätigkeit und Projekte informieren. Eine planbare Zusicherung dieser Gelder ist jedoch nicht möglich.

Die Gründung einer gemeinnützigen Gesellschaft mit beschränkter Haftung $(\mathrm{gGmbH})$ entwickelt sich zu einer beliebten Alternative gegenüber der Vereinsgründung. Insbesondere im professionellen Dienstleistungsbereich mit gemeinnützigen Zielen bietet die gGmbH kürzere Entscheidungswege über eine hauptamtliche Geschäftsführung anstelle einer Mitgliederversammlung im Verein. Eine Gründung kann auch mit weniger als 25.000,00 Euro Stammkapital erfolgen: Mit einem Kapital von mindestens 1,00 Euro wird eine gemeinnützige Unternehmergesellschaft (haftungsbeschränkt) notariell beglaubigt und in das Handelsregister eingetragen. Diese Form hat die gleichen Rechte und Pflichten wie eine $\mathrm{GmbH}$ und soll den Weg zur Gründung erleichtern. Die Umwandlung vom Verein in eine $\mathrm{gGmbH}$ ist derzeit zunehmend bei Kindertageseinrichtungen in Deutschland zu beobachten.

\section{Fazit}

Die Finanzierungswege psychomotorischer Angebote sind vielfältig. Kinder, Jugendliche und Erwachsene, die in ihrer Entwicklung und gesellschaftlichen Teilhabe beeinträchtigt oder davon bedroht sind, haben einen gesetzlichen Anspruch auf rehabilitative Maßnahmen. Die Beantragung der Kostenübernahme gestaltet sich im therapeutischen Bereich in der Regel mit einem Mehraufwand für die Familie wenig nie- derschwellig und dauert mitunter mehrere Wochen. Eine Alternative bietet die unabhängige, jedoch für viele Familien nicht leistbare Privatfinanzierung. Der sich zurzeit entwickelnde präventive Bereich wird überwiegend noch privat finanziert und nur in wenigen Einzelfällen von den Krankenkassen übernommen. Die Beantragung von Drittmitteln ist für die Planung und Durchführung gemeinnütziger Projekte zur Psychomotorik ein wesentlicher Bestandteil der Arbeit im präventiven Bereich. Hier bietet die Rechtsform der gemeinnützigen Gesellschaft mit beschränkter Haftung eine gute Alternative zur Vereinsarbeit.

\section{Caterina Schäfer}

DOI 10.2378/motorik2016.arto8d

\section{Anzeige}

\begin{tabular}{|} 
"Zusatzqualifikation Psychomotorik“ \\
Ein heißer Monat Juni 2016! \\
Denn dann beginnen unsere neuen Weiterbildungsreihen \\
"Zusatzqualifikation Psychomotorik“ \\
200-stündig, berufsbegleitend, in \\
• Berlin Frankfurt/M • München \\
1./2. April 2016 das IBP-Symposion zum Thema \\
"Psychomotorik in Verbundenheit“ \\
- tanzen, singen, spielen, lachen - \\
in Gröbenzell bei München \\
www.ibp-psychomotorik.de
\end{tabular}

